\title{
Le dualisme comportemental des agriculteurs : une interprétation institutionnaliste dialectique
}

Denis Barthélémy, Jean-Pierre Boinon et Martino Nieddu

\section{(2) OpenEdition \\ 12 Journals}

Édition électronique

URL : http://journals.openedition.org/ei/777

DOI : $10.4000 /$ ei. 777

ISSN : 2553-1891

Éditeur

Association Économie et Institutions

Édition imprimée

Date de publication : 1 décembre 2003

Pagination : 119-150

ISSN : 1775-2329

\section{Référence électronique}

Denis Barthélémy, Jean-Pierre Boinon et Martino Nieddu, « Le dualisme comportemental des agriculteurs : une interprétation institutionnaliste dialectique », Économie et institutions [En ligne] 3 | 2003, mis en ligne le 31 janvier 2013, consulté le 20 avril 2019. URL : http://

journals.openedition.org/ei/777 ; DOI : 10.4000/ei.777 


\title{
Le dualisme comportemental des agriculteurs : une interprétation institutionnaliste dialectique
}

\author{
Denis Barthélémy, INRA-MONA \\ Jean-Pierre Boinon, UMR INRA-ENESAD en Economie et Sociologie \\ Rurales \\ Martino Nieddu, ESSAI-OMI, UFR Sciences économiques et de gestion
}

Des écarts importants aux normes de l'économie de marché sont très fréquemment observés dans le secteur agricole. Le fait que de tels écarts se reconstituent en permanence, dans le domaine des marchés des produits aussi bien que de ceux des moyens de production, malgré les efforts d'institutions diverses (Nieddu, 2002) est souvent analysé à partir de considérations sur l'irrationalité supposée des acteurs ou sur leurs comportements opportunistes et leur capacité à mobiliser les subventions publiques grâce à des pratiques de lobbying condamnables (Bergmann, Baudin 1989, Pondaven 1989). Or il est possible d'envisager une autre approche, où de tels écarts seront reconnus comme le signe d'une rationalité économique qu'il convient d'expliciter: on adopte alors une démarche compréhensive qui nécessite d'ordonner les faits selon une cohérence qui n'est pas donnée à priori, et qui doit être découverte au cours de l'étude.

L'agenda institutionnaliste permet selon nous de développer une telle approche ${ }^{1}$. Nous nous proposons donc de mettre en regard les résultats de travaux que nous avons conduits sur les processus d'allocation des moyens de production dans l'agriculture française ${ }^{2}$, avec les approches dite "vieil institutionnalisme " qui connaissent aujourd'hui une réévaluation importante (Bazzoli, 1999). Une telle mise en regard nous amène à souligner l'intérêt de deux points clés de l'approche de Commons : la notion de transaction comme unité de base de l'analyse économique, et celle d'institution qui lui est liée ; la formation d'une valeur sociale raisonnable comme produit d'un

1 Une première version de ce travail a été présentée aux journées "Institutionnalismes et Evolutionnismes " de Lyon en décembre 2002. Nous remercions vivement les referees anonymes qui nous ont suggéré avec justesse des modifications et des développements. Au fil de ce texte l'ensemble des citations en langue anglaise ont été traduites par nousmêmes.

2 Voir en bibliographie divers documents publiés par Barthélemy et alii, Gaignette et Nieddu, Nieddu, qui concernent ce champ d'investigation aussi bien que celui des prix des produits.

118 Economie et Institutions $-n^{\circ} 3-2^{e}$ semestre 2003 
processus social d'évaluation contradictoire, plutôt que de la renvoyer à un modèle abstrait de marché.

"Question agraire " contemporaine et institutionnalisme

On s'appuiera pour ce faire sur un aspect essentiel en agriculture : le caractère familial de l'activité agricole, régulièrement dénoncé, depuis la seconde moitié du XIXème siècle, comme un archaïsme appelé à disparaître. L'exploitation agricole aurait nécessairement dû adopter le modèle de l'entreprise industrielle, qui sépare l'activité productive des relations familiales et le capital de l'entreprise du patrimoine de la famille, pour des motifs d'efficience marchande. Ce débat, connu sous le nom de "question agraire " depuis Kautsky s'est trouvé périodiquement réinvesti en raison de la résistance qu'opposaient les faits à la théorie : l'exploitation familiale persiste comme forme d'organisation dominant, encore aujourd'hui, le secteur agricole (Gasson, Errington, 1993). La perpétuation de cette forme de production a principalement été vue comme l'effet de divers mécanismes institutionnels devant être condamnés en tant qu'encouragement à l'inefficience marchande (Bergmann, Baudin, 1989; Tracy, 1994).

Pourtant, on pourrait envisager le questionnement théorique différemment. L'économie de marché ne naît pas spontanément (Polanyi, 1944). Il s'agit au contraire d'un processus destiné à instituer l'efficacité marchande comme principe normatif, comme règle modelant comportements et choix cognitifs dans le secteur considéré. Une telle institutionnalisation ne peut créer ex abrupto le nouvel ensemble de normes de comportement. Il faut donc plutôt envisager une transformation de la situation préexistante, ainsi que des compromis en fonction des résistances rencontrées qui tient à la consistance et à la configuration des relations économiques préexistantes ${ }^{3}$.

3 On a montré ailleurs que l'efficacité marchande ne va pas de soi, au point que la construction institutionnelle mise en œuvre en France et en Europe dans les années 1960 peut précisément s'expliquer par le fait que les paysans faiblement insérés sur les marchés étaient somme toute peu sensibles au critère d'efficacité marchande. Il a fallu alors trouver des dispositifs institutionnels pour les éliminer (Gaignette \& Nieddu, 1994). Au nom de l'efficacité marchande, on a dû mettre en place des institutions non marchandes et un droit rural exorbitant du droit commun, pour réussir à sélectionner les agriculteurs censés correspondre à cette efficacité, grâce à des normes portant sur les produits (et de leur qualité, ou plutôt absence de qualité à l'époque), et sur les arrangements organisationnels (obligations d'adhésion à un groupement de producteurs, distribution administrée de bonification de crédit, etc.). 
Un tel problème n'était pas traité dans les quatre grands types d'approches analytiques mises en oeuvre: (1) Certains rejetaient la dimension familiale de l'agriculture hors de l'analyse économique, ce qui les conduisait soit à exiger arbitrairement de ceux-ci qu'ils excluent les finalités familiales de leur activité d'entreprise (Chombart de Lauwe et al. 1969), soit à dénoncer leur irrationalité (Bergman, Baudin 1989, Mahé et Ortalo-Magné 2001), (2) un point de vue de gestionnaire acceptait la variété des objectifs pour l'entreprise, aussi bien familiaux que marchands, mais sans s'interroger sur la consistance de la liaison entre ceux-ci (Brossier, et al.,1997), (3) d'autres se livraient à une surinterprétation en vue de dénoncer l'action lobbyiste des agriculteurs (Pondaven,1989), (4) d'autres encore, plus subtilement, proposaient la réduction de la rationalité familiale à la rationalité marchande selon une démarche lancastérienne et beckerienne (Caillavet et al. 1994).

Selon nous, ces approches présentent deux points communs. D'abord, elles partent d'un point de vue de normativité de l'action pour rendre compte des modes de coordination présidant à la configuration de l'exploitation agricole. Ensuite, ce point de vue est développé en étant indifférent à la place et au jeu des institutions. Or l'économie agricole se caractérise par une évolution permanente de son système d'allocation des moyens de production, à mesure de poussées et de contre-poussées exprimées par des lois, règlements, décrets, coutumes, qui se complètent, se divisent ou s'opposent les uns aux autres.

\section{Méthode abductive et formation d'une hypothèse explicative}

A l'opposé de ces points de vue en termes de normativité de l'action, nous nous sommes efforcés de reprendre les fondements de la méthode abductive, telle que le philosophe pragmatiste C.S. Pierce, qui a fourni le substrat du vieil institutionnalisme la définit : "Dans l'abduction, nous passons de l'observation de certains faits $\grave{a}$ la supposition d'un principe général, qui s'il était vrai, expliquerait que les faits soient tels qu'ils sont. Nous concluons donc à l'existence de quelque chose qui est totalement différent de tout ce que nous avons pu observer empiriquement, bref à quelque chose qui est dans la majorité des cas inobservable" (Pierce, cité par Guillemin, 1998, p.319). On peut alors décomposer la mise en œuvre de la méthode adductive en trois phases (1) la mise en ordre de faits stylisés paradoxaux, qui vont être reconnus comme incontournables et devoir être expliqués, (2) la mise en forme d'une hypothèse explicative s'appuyant sur les acquis théoriques du courant régulationniste, (3) la mise en ouvre d'une stratégie d'observation empirique de grandes régularités en rapport avec ces faits stylisés. La crédibilité de cette hypothèse explicative tient dans ses qualités heuristiques, sa 
capacité à mettre en forme, à donner à voir une réalité, qui, si elle existe bien en dehors de l'observateur, ne se donne à voir de façon intelligible que si l'on en construit une représentation.

L'hypothèse que nous proposons de développer est la suivante: de fait, on peut constater que les caractéristiques de l'exploitation agricole évoluent au cours du temps, sur les deux plans des relations marchandes et des relations patrimoniales. La famille se "modernise ", le statut de la propriété foncière agricole change, afin de laisser place aux exigences de la professionnalisation et de l'entreprise. En contrepoint, l'entreprise, au sens de la théorie de la firme, bénéficiant de cette évolution des règles patrimoniales ${ }^{4}$, se développe dans un contexte de concurrence marchande, ceci se traduisant par l'élimination des petites exploitations, l'accroissement de dimension et la rationalisation marchande de celles qui subsistent. Mais il est à remarquer que, au grand dam des contempteurs de l'agriculture familiale, le processus institutionnel qui sollicite et accompagne ce changement conserve rigoureusement les termes d'une relation entre un univers patrimonial, qui change progressivement dans ses contenus et ses titulaires, en sorte de s'ouvrir à l'entreprise, et une entreprise dont le développement est toujours soigneusement maintenu sous la dépendance d'une finalité patrimoniale. L'exploitant agricole, en tant qu'agent économique, manifeste un dualisme comportemental se référant selon les circonstances à deux logiques: une logique de comportement d'entrepreneur capitaliste, une logique de comportement patrimonial (de type familial d'abord, professionnel ensuite).

\section{Dualisme comportemental des agriculteurs et institutionnalisme}

Le défi posé à l'analyse est de prendre en compte un type de situation de double rationalité, l'objet de la description étant le statut des moyens de production de l'agriculture pris entre leurs insertions dans des relations marchandes et dans des relations patrimoniales. Il s'agit alors de décrire le mouvement relatif du capital et du patrimoine en agriculture dans une perspective institutionnaliste évolutive, comme le jeu réciproque entre l'institution marchande (centrée ici sur l'entreprise au sens de la théorie de la firme et les rapports sociaux correspondants) au sein de laquelle les moyens de production ont le statut de marchandise et de capital à accumuler, et l'institution patrimoniale (dont la famille est l'archétype, mais dont tout groupe social organisé selon des règles de solidarité et de

4 Abandonnant la réduction sémantique individualisante du terme patrimoine, réalisée à l'issue de la Révolution Française notamment, nous le reprenons dans un sens plus proche de son sens étymologique, comme dans l'expression apparue depuis une trentaine d'années de patrimoine commun (cf. Barrère et al, à paraître 2004).

Economie et Institutions $-\mathrm{n}^{\circ} 3-2^{\mathrm{e}}$ semestre 2003 
continuité est l'expression) dont le moyen est le patrimoine. Nous avons ainsi été conduits à organiser l'analyse en considérant le dualisme comportemental des agriculteurs, dont la mise en œuvre dépend du contexte auquel les acteurs se réfèrent, comme le moteur de la dynamique institutionnelle.

Dans ce domaine la tâche à accomplir comporte deux dimensions. L'une est de rapporter rigoureusement chaque norme à la valeur sociale qui lui correspond. L'autre consiste à mettre en relation les termes opposés pour proposer un schéma de lecture de la dynamique selon un jeu dans lequel l'institution de l'efficacité marchande se heurte à la résistance de l'institution patrimoniale préexistante, laquelle va être peu à peu transformée, évoluant vers de nouvelles formes de relations patrimoniales. En retour, l'ordre marchand en élaboration se trouve réduit dans ses prérogatives et infléchi dans ses orientations. En ce sens, notre recherche s'inscrit dans un schéma selon lequel "dans chacune de ces époques historiques de nouveaux concepts de droits et pratiques raisonnables ont rapidement supplantés les précédents, jusqu'aux concepts contestataires actuels de valeur raisonnable, dans un monde qui hérite de l'ancien mais est provoqué par les ajustements économiques imparfaits à un nouveau futur issu de l'obsolescence du passé " (Commons, 1934, p. 682).

Dans une première partie, notre hypothèse interprétative servira à conduire et ordonner la description empirique qui portera essentiellement sur la caractérisation des relations de droit entre les acteurs, et leur évolution, selon la tradition mise en exergue par les tenants du "vieil institutionnalisme ": "c'est dans le legal-economic nexus que se déterminent l'organisation et le contrôle du système économique" (Bazzoli, Kirat, 1997, 268), que s'expriment "les ajustements continuels opérés entre les règles juridiques et les processus économiques " (Kirat, 2001, 17). Dans la seconde partie, on mettra en regard les apports de cette description des singularités des processus d'allocation des ressources dans l'agriculture française ${ }^{5}$ avec les débats visant à définir l'approche institutionnaliste.

5 On se limite ici au cas français ; à travers des modalités empiriques un peu différentes, on trouve des situations analogues dans les autres pays (Barthélemy \& David, 2001, Barthélemy, 2002).

$122 \quad$ Economie et Institutions $-n^{\circ} 3-2^{e}$ semestre 2003 


\section{Première partie - Dynamique institutionnelle et développement de l'exploitation agricole}

Dans son archétype, la famille représente une institution orientée vers la continuité intergénérationnelle. L'ensemble des biens familiaux forme patrimoine, leur usage actuel devant assurer la perpétuation du groupe familial, tandis que leur aliénation est exclue ou doit rester exceptionnelle. Reposant sur de tels principes, l'exploitation familiale n'est pas naturellement portée vers le marché, qui implique aléas et risques de perte ; la notion d'entreprise lui est extérieure et opposée ${ }^{6}$. L'évolution de l'exploitation familiale vers l'idéal-type de l'entreprise nécessite une série de ruptures de l'ordre patrimonial dans ses dispositifs.

\section{1- L'altération du fonctionnement patrimonial traditionnel en faveur d'un développement d'entreprise}

\subsubsection{Détourner l'héritage}

La législation française, influencé par la volonté révolutionnaire d'éradiquer les fondements patrimoniaux de l'Ancien Régime, organise dans la fratrie le partage égalitaire des biens en nature ${ }^{7}$. Ce droit égalitaire est dénoncé dès le $19^{\circ}$ siècle comme une "machine à hacher la terre ", tant il conduit à un morcellement des parcelles. Pour pallier ce risque de déstructuration périodique, le décret-loi du 17 juin 1938 énonce la possibilité que la formation des lots, lors du partage des héritages, évite la division des exploitations et établit le droit pour l'un des héritiers de revendiquer dans son lot l'attribution préférentielle de l'exploitation agricole à la mise en valeur de laquelle il a participé.

On met donc l'un des héritiers en position de s'opposer aux autres, au nom de la préservation de l'intégrité d'une "unité économique", entité juridique nouvelle qui surgit au sein du patrimoine et de manière contradictoire à celui-ci : l'unité économique se réfère au marché, tandis que le patrimoine relève d'une conception extérieure à celui-ci ${ }^{8}$. Ce dispositif s'est trouvé par

6 On trouvera une description de l'incompatibilité des règles de solidarité de l'économie paysanne traditionnelle et de celles de l'économie de marché in : Georgescu-Roegen, 1965.

7 Conformément à la méthode du "vieil institutionnalisme ", nous prenons un point quelconque comme origine du temps de l'analyse, c'est-à-dire un état déjà transformé des relations patrimoniales. Hypothétiquement, on pourrait se réfèrer à la Cité Antique (Fustel de Coulanges, 1864) comme modèle de fonctionnement patrimonial "pur ".

8 Et en même temps, on incite ou on oblige à ne pas respecter le partage en nature, en imposant une égalité des lots selon leur valeur marchande, alors 
la suite renforcé. L'attribution préférentielle de 1938 suppose une indemnité des co-héritiers à la valeur vénale, or le coût des soultes dues par le bénéficiaire peut compromettre la poursuite de l'exploitation. La loi du 4 juillet 1980 établira que le demandeur peut soumettre le partage de l'indivision successorale à la conclusion d'un bail en sa faveur de la part de ses cohéritiers. Autrement dit, soit il demeure gérant de fait du bien par nécessité de sauvegarde de l'unité de production, soit il obtient gratuitement un bail qui lui garantit une position hégémonique sur l'exploitation.

\subsubsection{Imposer l'investissement au patrimoine foncier}

L'instauration d'un statut du fermage et du métayage, en 1946, participe de la régression de la logique patrimoniale: le locataire d'un bien foncier agricole est doté de la capacité d'imposer au propriétaire le respect d'une normalité d'entreprise. Depuis le $18^{\circ}$ siècle les praticiens des baux ruraux relevaient qu'accroître la production nécessite de dépenser en moyens de production dont la durée d'existence peut excéder celle du bail. Le locataire a donc besoin de garantie de stabilité, ou de remboursement de la valeur résiduelle de ses investissements en cas de départ. Le propriétaire n'est pas nécessairement dans une disposition favorable à cet égard. Lorsque, comme fréquemment, sa propriété lui vient de l'héritage, la terre n'est pas une ressource à risquer dans l'activité de production pour le marché. En déléguant la culture du sol au fermier, il ne confie à celui-ci que le soin de recueillir "tous les fruits de l'héritage affermé" selon l'expression toujours présente de l'article 1774 du Code Civil. Il répugne à se laisser impliquer par les dépenses d'investissement du fermier.

A partir de 1946, la législation du fermage l'y oblige. Le fermier bénéficie d'une durée minimale de 9 ans pour son bail, indéfiniment renouvelable sauf reprise personnelle du propriétaire, du droit de choisir le mode de culture et de réaliser des investissements avec une certaine liberté, assortie de l'obligation pour le propriétaire de les indemniser en fin de bail. Ces différentes dispositions ne cesseront d'être réactualisées et étendues par la suite, dans le sens d'une plus grande liberté d'investir du fermier (lois de 1967, 1970, 1975). Dans le conflit entre agriculture patrimoniale, passéiste et routinière, et agriculture d'entreprise, le locataire est placé en position de promouvoir l'entreprise. L'enjeu est clairement l'entreprise, et non le fermier : le propriétaire conserve la possibilité de reprendre l'usage de son bien, à condition de s'engager

même que le fonctionnement patrimonial normal interdit de constituer l'hypothèse d'une mise en vente.

124 Economie et Institutions $-n^{\circ} 3-2^{e}$ semestre 2003 
à cultiver lui-même pendant 9 ans "selon les usages de la région, en fonction de l'importance de l'exploitation" (Code Rural, art. L 411-59).

\subsubsection{Promouvoir la rationalité d'entreprise}

A la suite du Traité de Rome qui, par la Politique Agricole Commune, ouvre des perspectives d'expansion à l'agriculture française, les lois d'orientation agricole de 1960-62 se donnent pour objectif de promouvoir une "exploitation agricole viable". Cette notion se réfère à une "utilisation rationnelle des capitaux et des techniques" (Loi 60-808, art.7), par où il faut entendre que de telles unités économiques doivent permettre à "la main d'œuvre familiale et non familiale" de recevoir "une rémunération $d u$ travail correspondant à celle qu'elle aurait pu obtenir dans les autres activités susceptibles de l'employer", et "aux capitaux fonciers et d'exploitation "d'obtenir " un intérêt convenable " (art. 8).

Là où la conception patrimoniale voit la liaison indissoluble des personnes et des biens dans l'exploitation agricole, la loi distingue une main d'œuvre éventuellement transposable dans n'importe quel secteur d'activité, et attribue aux biens qu'elle conçoit séparés des personnes, le statut d'un capital devant être rémunéré selon les conditions du marché financier, c'est-à-dire implicitement définis comme aliénables au gré de leur productivité marchande. Cette redéfinition n'est pas seulement théorique: des moyens spécifiques sont mis en place en vue de favoriser l'émergence d'une telle entité : sociétés d'intervention sur le marché foncier (SAFER) ; instauration d'un contrôle administratif du droit d'exploiter (contrôle des structures); aides à l'installation et au développement (prêts bonifiés, dotations...) ; d'importants moyens financiers sont mis en place (indemnités viagères de départ) pour inciter à la cessation d'activité des exploitations non viables, en vue de rendre disponibles des surfaces pour les exploitations viables.

La loi classe ainsi les exploitations: d'un côté celles qui, aujourd'hui ou bientôt, répondent aux critères de viabilité marchande, synthétiquement exprimée par une surface minimale, et qui bénéficieront des moyens de protection et de soutien mis en place, et de l'autre celles qui, étant estimées non viables, ne seront pas aidées et pourront être démembrées. Ainsi, la politique agricole des années 60 vise à généraliser à l'agriculture tout entière le projet d'entreprise. Ce nouveau cours de la législation et de la politique agricole aura un impact tel que l'économie de marché et l'entreprise paraissent alors avoir triomphé en agriculture, annihilant l'économie patrimoniale. Nombreux sont ceux qui l'ont perçu ainsi, annonçant à grand fracas la fin de l'agriculture familiale (Gervais et al, 1965), sans voir que se constituaient simultanément des limites au développement de l'entreprise. 


\section{I.2- Un développement d'entreprise bridé par la finalité patrimoniale}

\subsubsection{Englober l'entreprise dans la famille}

Le contrôle administratif des "structures agricoles " comporte en réalité deux seuils. En même temps que le seuil minimum de viabilité, formant la frontière entre exploitations découragées et exploitations aidées, il établit un seuil maximal au-delà duquel l'autorisation d'exploiter peut être refusée. L'objectif de la loi est de "promouvoir et favoriser une structure d'exploitation de type familial " (Loi 60-808, art.2), représentée par des exploitations mises en valeur par " deux unités de main d'oeuvre " (art. 7).

L'organisation d'une protection et l'attribution de moyens à l'exploitation viable ne concernent celle-ci que pour autant qu'elle demeure, malgré tout, une entité familiale. La rupture du lien patrimonial, par laquelle les exploitants familiaux sont considérés comme une main d'œuvre et les moyens de production ainsi que la terre comme capital, n'est postulée que dans le souci de discriminer entre les exploitations viables et non viables au sens marchand. Mais se trouve aussitôt rétabli, au sein de l'ensemble des exploitations dites viables, le principe du lien qui unit les personnes et les biens, par le freinage de la croissance de l'entreprise, dès lors que la surface d'exploitation dépasserait la limite supposée d'une structure familiale ${ }^{9}$.

\subsubsection{Assigner une dimension intergénérationnelle à l'entreprise}

Plus radicalement, une autre notion se développe à partir des années 1970 : l'installation des jeunes ${ }^{10}$. Installer un jeune signifie faire de lui un chef d'exploitation, c'est-à-dire, selon le concept de l'entreprise englobée dans la famille, le détenteur de la propriété de l'ensemble ou d'une fraction significative des actifs de l'entreprise. Un tel objectif est dépourvu de sens en terme de rationalité d'entreprise, car selon celle-ci la propriété d'entreprise revient à qui offre le meilleur prix, et sans doute pas à un jeune dépourvu

9 La détermination du caractère familial de l'exploitation par la limitation de la quantité de main d'œuvre ne représente qu'une dimension du processus institutionnel mis en oeuvre. La propriété directe du capital ou d'une part majoritaire de celui-ci en est une autre, comme on peut le voir dans le régime juridique spécifique des sociétés d'exploitation agricole.

10 A l'origine se trouve une politique européenne en faveur du renouvellement des générations en agriculture : la Directive CEE du 17 avril 1972 prévoit la possibilité d'attribuer une dotation aux jeunes qui s'installent.

126 Economie et Institutions $-n^{\circ} 3-2^{e}$ semestre 2003 
d'argent ${ }^{11}$. Il s'agit donc de subvertir la logique marchande pour imposer une finalité de type patrimonial.

Traditionnellement, les exploitants agricoles ont eu coutume de préserver l'unité de l'exploitation agricole en la transmettant à l'un de leurs descendants ${ }^{12}$. A l'encontre toutefois des prescriptions du Code Civil, ils se référaient au principe d'une solidarité familiale maintenue, l'un d'entre eux recevant l'exploitation agricole sous condition implicite de prise en charge des parents âgés, et éventuellement des frères et sœurs dans le besoin. Cette pratique, très générale dans l'agriculture (David, 1986 ; Daucé et al., 1993), opère souvent par un glissement subreptice de la propriété de l'exploitation entre le père et son successeur, en contravention aux règles formelles des donations et transmissions dans les pays de partage successoral égalitaire. Elle devient plus difficile à mettre en œuvre lorsque l'entreprise se structure (impliquant une comptabilité) et que la valeur marchande des actifs de l'entreprise ne cesse de s'accroître, rendant plus problématique l'acceptation tacite de la fratrie. Et en même temps le problème a pris une autre dimension : s'agissant d'une entreprise, le jeune doit l'acheter afin de s'inscrire pleinement dans les rapports marchands et professionnels correspondants ; il ne peut plus être là simplement comme fils de son père, mais doit se présenter comme jeune professionnel acheteur de l'entreprise. Ainsi l'héritage doit être relégué dans l'univers familial maintenant déprofessionnalisé (même si le jeune obtient des ressources sur sa part d'héritage); il doit acheter l'entreprise. Une telle gageure conduirait à l'impasse si, fort opportunément, la loi n'offrait une solution, précisément parce qu'elle fait du bail rural un objet possédant en lui-même une dimension patrimoniale, en le rendant incessible en dehors de la famille et interdit de valorisation marchande.

Le bail rural est en effet défini comme un contrat intuitu personae, par où il est incessible. Cette interdiction de cession connaît une exception notable, en faveur du descendant ou du conjoint du locataire (art. L 411-35 CR). Une telle dérogation à l'intuitu personae ne peut se comprendre qu'en référence à l'ordre patrimonial, qui se caractérise par un continuum entre les membres

11 Et certains dénoncent comme contraire à l'efficacité marchande toute aide à la transmission d'entreprise (Papillon, 1995).

12 La perpétuation simultanée de la famille et de la ferme a de tout temps posé problème en agriculture, conduisant à différents types de solutions. On trouvera une étude particulièrement riche des systèmes de règles juridiques et de coutumes caractérisant les diverses périodes et régions de l'Europe in : Augustins, G., 1989. La pratique qui est décrite ici trouve des racines très anciennes, mais elle est envisagée dans sa novation, à l'époque de l'émergence d'une entreprise en agriculture et de l'extension des équivalences marchandes que celle-ci implique. 
du groupe, impliquant par exemple une succession des générations dans la propriété du patrimoine sans espace de négociation. Ainsi le fils peut se trouver substitué à son père vis à vis du propriétaire foncier, selon les principes d'une transmission patrimoniale, dont le caractère non marchand est solennellement confirmé par l'article L 411-74 qui interdit l'affectation d'une valeur vénale du bail13.

Ainsi, l'installation des jeunes en agriculture s'inscrit apparemment dans une normalité marchande, puisqu'ils achètent l'entreprise. Mais dans la grande majorité des cas (les cessions familiales), l'acquéreur se trouve désigné avant la mise en marché, si bien que le prix est ajusté en sorte de pouvoir être payé sur les moyens dont dispose l'acheteur-descendant. La cession de bail consentie par les parents s'opère selon les termes de la loi, c'est-àdire gratuitement, tandis que les autres actifs sont cédés à une valeur déterminée de manière telle qu'elle corresponde à ses droits d'héritage, augmentés de la dotation d'installation versée par l'Etat et des emprunts à taux bonifiés dont il peut bénéficier.

L'imposition à l'entreprise d'une finalité de continuité intergénérationnelle se réalise ainsi par une négociation marchande altérée : celle-ci combine d'une part les aides de l'Etat et les apports liés à l'héritage familial, en sorte de placer les jeunes dans une position d'acquéreurs privilégiés lors des cessions d'entreprise, et d'autre part la mise hors du marché de l'un des principaux actifs de l'exploitation (le bail sur les terres) en le dotant d'un statut patrimonial.

\section{I.3- La formation de nouvelles relations patrimoniales}

Le mouvement précédemment décrit comporte l'amoindrissement des relations patrimoniales familiales préexistantes, en sorte de faire place à des relations d'entreprise, et le maintien du développement de celles-ci dans une limite telle qu'elles ne puissent aller jusqu'à renverser toute perspective patrimoniale. Le mouvement vers l'entreprise se trouve ainsi combiné à (et borné par) l'établissement de nouvelles relations patrimoniales, plus favorables aux caractéristiques de l'entreprise sans épouser sa rationalité, qui prennent le relais des relations familiales en déclin. Un nouveau patrimoine émerge, ayant perdu les caractéristiques familiales précédentes pour s'enraciner désormais dans le groupe professionnel. Ceci engage la définition de règles d'appartenance des personnes à ce groupe, l'établissement de biens patrimoniaux qui lui soient propre, ainsi que des valeurs économiques patrimoniales correspondantes.

13 A la différence du bail commercial, industriel ou artisanal, qui représente de droit un actif marchand incorporel monnayable. 


\subsubsection{Constitution d'un groupe professionnel, établissement de biens patrimoniaux et de règles d'allocation patrimoniales.}

L'appartenance au groupe des exploitants agricoles s'établit à partir de la définition des modalités d'accès à cette profession. On trouve ainsi exposés des critères de compétence professionnelle et de viabilité de l'exploitation (au sens précédemment décrit) à l'occasion de l'installation d'un jeune agriculteur bénéficiant des aides de l'Etat (Code Rural, art. R 343-4 et R 343-5). Des caractéristiques du même ordre sont nécessaires lorsqu'un propriétaire foncier veut reprendre les terres qu'il donne en location pour les cultiver lui-même (art. L 411-59 et $\mathrm{R}$ 331-1 CR) (c'est la seule hypothèse pour qu'il puisse évincer le fermier) ou lorsque le descendant d'un fermier souhaite bénéficier de la cession du bail de son père.

Parallèlement à la constitution du groupe professionnel, un ensemble de biens impliqués dans l'activité professionnelle prend une consistance patrimoniale. Cela signifie qu'ils sont réservés à l'usage des professionnels, et que leur répartition entre ceux-ci échappe aux lois du marché. Font partie de ces biens patrimoniaux le bail rural, ainsi que le droit d'exploiter les terres et les droits à produire.

Pour ce qui est du bail rural, on a déjà indiqué que la cession du bail à un descendant exigeait de ce dernier des aptitudes professionnelles et financières, de même que l'on a souligné que le refus de renouvellement du bail par le propriétaire nécessitait que celui-ci s'engage à cultiver personnellement, dans des conditions de professionnalité définies. Le bail rural se trouve ainsi inséré dans une double limitation qui en fait un bien réservé aux professionnels de l'agriculture.

Le droit d'exploiter résulte du contrôle des structures : il ne suffit pas d'avoir acheté ou loué une terre pour pouvoir l'exploiter, il faut avoir obtenu une autorisation qui, en tant que telle, représente un nouveau droit, non marchand, réservé aux professionnels de l'agriculture selon le concept d'exploitation familiale viable. L'existence d'une telle forme de droit n'a en soi rien d'extraordinaire, on en trouve l'analogue avec le droit de construire sur une propriété Ce sont ses conditions d'exercice qui doivent être remarquées: extériorité à l'univers marchand puisqu'il ne peut pas être négocié, règles spécifiques de réservation et de répartition entre les seuls professionnels de l'agriculture ${ }^{14}$.

14 De ce point de vue, le droit d'exploiter joue un rôle de renforcement de la non négociabilité du bail rural, puisque la conclusion d'un bail n'a d'intérêt que si le bénéficiaire est susceptible de se trouver en conformité avec la réglementation des structures. Et symétriquement d'ailleurs, un propriétaire 
Quant aux droits à produire, selon le terme imposé par l'usage, il s'agit des instruments de soutien à l'agriculture familiale mis en place par la Politique agricole commune à partir des années 80: quotas et droits à prime. Ils forment aujourd'hui une part substantielle des moyens de production de l'agriculture car ils concourent à la formation de plus de la moitié du revenu des exploitations. A l'origine, ils sont attribués aux producteurs en place, et non mis aux enchères, comme ils devraient l'être en bonne logique marchande. En France, leurs transferts marchands entre producteurs sont en outre soit empêchés, soit altérés par des règles patrimoniales ${ }^{15}$.

\subsubsection{Des règles patrimoniales d'allocation}

Ces divers biens patrimoniaux, le bail rural, le droit d'exploiter, les droits à produire, sont en effet soumis à des règles positives de répartition selon des critères d'économie patrimoniale :

- Le bail rural est de droit incessible, si ce n'est dans un cadre familial: son allocation doit satisfaire aux besoins de continuité transgénérationnelle de l'exploitation.

- Le droit d'exploiter est attribué par le jeu des seuils aux exploitants viables (sous la double dimension, marchande et patrimoniale du terme). Concrètement, cela signifie que sont privilégiés les exploitants moyens et/ou les jeunes désirant s'installer.

- Pour les droits à produire liés aux surfaces, la répartition passe par le canal d'un contrôle analogue à celui des structures, dont il vient d'être question. Les quotas laitiers et droits à prime sont soumis lors des transferts à des mécanismes de prélèvement spécifiques au détriment des plus gros exploitants, donnant lieu à restitution en faveur des moyens exploitants et/ou des jeunes qui s'installent (Barthélemy, David, 2001 ; Barthélemy, 2002).

$\mathrm{Au}$ total, cette gestion administrée ${ }^{16}$ suit un programme d'allocation des biens particulièrement clair. Sans supprimer le

qui désire effectuer une reprise de son bien pour exploitation directe doit satisfaire aux exigences du contrôle des structures (art. L 411-58 CR), outre celles de capacités professionnelles.

15 Il en va de même, à des degrés divers et selon des règles spécifiques, dans les différents pays européens (Barthélemy, David, 2001).

16 Pour faire court, car si l'administration est en titre responsable de ces actions de contrôle, prélèvement et répartition, elle partage de fait le pouvoir avec une Commission Départementale d'Orientation Agricole (CDOA) principalement représentative des exploitants agricoles. L'expression la plus correcte serait de dire qu'il y a gestion des biens professionnels par le groupe professionnel, sous le contrôle de l'administration (Barthélemy, 2002 ; Berriet-Solliec, Boinon, 2002).

130 Economie et Institutions $-n^{\circ} 3-2^{e}$ semestre 2003 
marché, il en limite les effets, au bénéfice de règles patrimoniales qui freinent le développement des entreprises tendant à excéder la norme du groupe professionnel des exploitants familiaux. Autrement dit, il s'agit d'appliquer des règles de solidarité inter- et intragénérationnelle, fondement même d'une économie patrimoniale.

\section{I.4- Prix patrimoniaux versus prix marchands}

Dans le développement de ces nouvelles relations patrimoniales de type professionnel, qui prennent le relais des relations familiales, une étape importante est l'élaboration d'un référentiel de prix patrimoniaux, distincts des prix de marché.

\subsubsection{L'établissement des prix patrimoniaux}

La pluralité des biens patrimoniaux, la complication des règles qui leur sont à chacun appliqué, posent le problème de l'opérateur qui mesure les équivalences entre droits, et donc qui, in fine, explicite les prix patrimoniaux qui leur sont attachés.

En France, les Commissions Départementales d'Orientation Agricoles (CDOA) contrôlent simultanément les droits d'exploiter et les droits à produire depuis 1995. Pour ce qui est des droits d'exploiter, il s'agit de les accorder ou de les refuser aux candidats à l'exploitation qui le demandent, selon que l'exploitation résultante respecte ou non l'intervalle de surface représentatif de "l'exploitation familiale viable". Pour ce qui concerne les droits à produire, après qu'ils aient été prélevés lors des transferts aux exploitants dépassant certains seuils de quantités de droits, ils doivent être réattribués à des exploitants jugés prioritaires. Ces commissions ont donc à répartir des biens patrimoniaux (en tant qu'ils sont répartis en dehors du marché, selon des principes de solidarités) hétérogènes entre eux. Elles en sont venues à établir des équivalences destinées à exercer la cohérence de leurs actions. Lorsque les CDOA ont à prendre position sur une demande d'autorisation d'exploiter des surfaces, elles doivent examiner les quotas et droits à prime déjà détenus par le demandeur, ou qu'il obtiendra avec les surfaces pour lesquelles il demande cette autorisation; et lorsque des attributions de droits à produire sont effectuées à partir des quantités prélevées sur les transferts, on leur suggère d'établir une comptabilité permettant de comparer l'impact relatif des attributions, selon leurs natures, sur le revenu des bénéficiaires ${ }^{17}$.

17 Loi du $1^{\circ}$ février 1995 (son article 15 indique que, lors des attributions de droits à produire "des équivalences peuvent être établies entre les références et les droits concernant des productions différentes en fonction du revenu procuré par ces productions ") ; loi du 9 juillet 1999 (son article 22 précise que dans l'attribution des droits d'exploiter des surfaces agricoles il faut

Economie et Institutions $-n^{\circ} 3-2^{e}$ semestre 2003 
Concrètement, les CDOA se fixent un objectif, sous la forme d'un niveau de revenu marchand souhaité pour une exploitation agricole utilisant une quantité de main d'œuvre familiale déterminée. Elles évaluent la contribution relative d'une unité de surface, d'un droit à produire, ou d'un type de production spéciale, à ce résultat. Elles sont ainsi à même de classer les exploitations demanderesses par rapport au standard de résultat fixé, ainsi que l'impact qu'aurait une autorisation de cultiver de nouvelles surfaces ou l'attribution de tel ou tel droit à produire (Roussel, 2001).

De tels choix traduisent les normes de production et dimensions d'exploitations que les commissions départementales entendent appliquer dans leurs ressorts, pour assurer au mieux l'allocation patrimoniale des ressources d'exploitation dont elles sont gestionnaires. Par ce système d'équivalence généralisée, les droits d'exploitation et les droits à produire se trouvent dotés d'un prix patrimonial spécifique, extérieur au marché, qui permet à chacun de savoir, compte tenu des crédits résultants de sa situation professionnelle spécifique, quelle surface il peut prétendre exploiter ou combien et de quelle catégorie de droits à produire il peut espérer bénéficier.

\subsubsection{Les prix marchands}

L'élaboration d'un système de prix patrimoniaux pour les moyens de production de l'agriculture ne fait pas pour autant disparaître leur prix marchand. Les relations d'échange marchand persistent, bien que leur espace d'exercice ait été amoindri. Ainsi, l'instauration du droit d'exploiter n'a pas supprimé le marché foncier qui assure le transfert du droit de propriété. Simplement il bloque la possibilité de cultiver lorsque la taille de celle-ci excéderait la plage consentie à l'activité d'entreprise. Dans cet intervalle, il y donc toujours un marché foncier et un prix de la terre en tant que capital foncier de l'entreprise.

La situation est un peu plus complexe en ce qui concerne le bail rural, car celui-ci est défini par la loi comme incessible et dépourvu de valeur pécuniaire. Il semble ainsi n'être doté que d'une nature patrimoniale. En fait, la contradiction créée par la double inscription des agriculteurs dans des relations patrimoniales et dans des relations marchandes est tellement forte que l'interdit légal est transgressé. Dans le cadre des cessions familiales, le principe de gratuité monétaire du bail est en général respecté. Mais dès lors que la cession d'exploitation s'opère entre non apparentés, le bail est

"prendre en compte les références de production ou droits à aide dont disposent déjà le ou les demandeurs ainsi que ceux attachés aux biens objets de la demande en appréciant les conséquences économiques de la reprise envisagée ").

132 Economie et Institutions $-n^{\circ} 3-2^{e}$ semestre 2003 
monnayé à la manière dont le bail commercial l'est dans les activités commerciales ou industrielles. Un marché occulte des baux se constitue $^{18}$, avec suffisamment de vigueur, de généralité et de consistance pour que la coutume s'impose et que la loi se trouve systématiquement contournée, dans les relations non familiales, même si les tenants d'une économie patrimoniale exclusive ne cessent de le dénoncer (Barthélemy, 1988).

A la différence du bail rural, il n'existe aucune interdiction légale d'attribuer une valeur pécuniaire aux droits à produire. Simplement, les textes par lesquels ils sont régis sont tels qu'aucun marché ne peut explicitement apparaître. Ainsi, de même que pour les baux ruraux, la tension entre l'absence d'instrument de marché spécifique et la logique d'entreprise conduit à des négociations marchandes pour les transferts qui se réalisent en dehors des relations familiales ou des procédures de prélèvement-attribution sous l'égide de la CDOA. S'agissant des droits à produire directement attachés à des surfaces désignées (primes céréalières et oléoprotéagineuses), ils sont transférés avec les surfaces correspondantes. Leur valeur marchande est alors incluse dans le prix de la terre sur le marché foncier, ou ajoutée à celui des baux négociés de façon occulte. Quant aux autres, et compte tenu des diverses réglementations spécifiques qui conduisent à ce qu'ils ne puissent être cédés indépendamment d'autres actifs de l'entreprise ${ }^{19}$, ils interviendront comme survaleurs de cheptels, bâtiments, matériels... (Barthélemy, David, 2001). Là aussi, ces pratiques de valorisation marchande sont régulièrement dénoncées par les tenants d'une économie patrimoniale exclusive.

\subsubsection{En conclusion: Conflit de valeurs, conflit de relations?}

La même terre agricole peut aussi bien être vendue au prix de marché que transmise gratuitement selon les normes de répartition de l'héritage. Le même bail et les mêmes droits à produire peuvent être soit cédés gratuitement à un descendant familial ou à un jeune non apparenté désirant s'installer, soit négociés à prix marchand à un agriculteur voisin désireux d'accroître son entreprise. Les mêmes biens se trouvent ainsi affectés de deux sortes de valeurs, chacune d'elle insérée dans une structure relationnelle spécifique.

18 Concrètement, le plus souvent, par surévaluation d'éléments d'actifs licitement vendus (bâtiments, matériels...).

19 Dans d'autres pays européens que la France, l'interprétation locale des règlements européens conduit à organiser des quasi-marchés ou de véritables marchés de ces droits à produire. Ils n'en sont pas moins tenus sous la domination d'un certain nombre de règles patrimoniales. (Barthélemy, David, 2001).

Economie et Institutions $-\mathrm{n}^{\circ} 3-2^{\mathrm{e}}$ semestre 2003 
Chaque agriculteur se trouve traversé par ce dilemme, en tant qu'il participe de chacun des ordres de relation. D'un côté, il est entrepreneur, inscrit dans l'économie de marché, ses relations d'échange et de concurrence; de l'autre côté, il participe au groupe patrimonial, familial et/ou professionnel, avec ses normes d'allocation solidaire. Le compromis institutionnel, qui règle les rapports entre ces deux formes d'économie, s'exprime de fait dans le dualisme comportemental de l'acteur : dans certaines circonstances, parce qu'il envisage son insertion marchande, il tend à privilégier les prix de marché et donc une valeur de marché, alors qu'à d'autres moments, il se réfère plutôt aux prix patrimoniaux et donc à une valeur de patrimoine.

Ce compromis n'est pas stable, en ce sens que, pour l'agriculteur, il n'est jamais pleinement satisfaisant: appliquer les valeurs de patrimonialité, c'est mettre en danger le développement de son entreprise; retenir les valeurs de marché, c'est œuvrer à la destruction du groupe familial et/ou professionnel. Cette ambivalence transparaît de fait dans la mise en œuvre par les agriculteurs du double référentiel de valeur auxquels sont soumis les ressources de l'activité agricole. Elle signifie que l'agriculteur n'additionne pas deux systèmes extérieurs l'un à l'autre, cherchant à combiner leurs rationalités ou à découvrir une méta-rationalité, mais qu'il est pris dans la nécessité de concilier deux référentiels qui se développent complémentairement et conflictuellement l'un à l'autre.

\section{Deuxième partie - Pour un institutionnalisme dialectique}

Le modèle d'interprétation comportementaliste des économistes repose sur l'hypothèse d'une fonction de préférence individuelle conduisant à l'élaboration de choix d'action dans un contexte particularisé de contraintes. Les caractéristiques que nous avons présentées des processus d'allocation des moyens de production de l'agriculture ne laissent guère prise à une telle voie d'interprétation. L'agent économique, ici l'agriculteur, est pris dans le réseau d'une double insertion comportementale : il est pour une face producteur marchand, et pour l'autre membre d'une communauté patrimoniale (tout d'abord familiale puis professionnelle). Quelle fonction de préférence lui attribuer ? L'ensemble des utilités dans la sphère de fonctionnement marchand est distincte ou même contradictoire à celui des utilités patrimoniales: se désigner producteur marchand, c'est-à-dire se référer à des valeurs d'individualisme et de concurrence, porte atteinte à l'appartenance patrimoniale, tandis que se définir comme membre d'une famille ou du groupe professionnel c'est adhérer à des valeurs d'égalitarisme et 
de solidarité directement contraires aux aspirations de l'être marchand. Quelle unité de comportement élaborer dans une telle situation d'opposition des préférences ?

L'impasse analytique de ce dualisme préférentiel et comportemental ne peut pas non plus être levée en imaginant la construction d'une méta-fonction de préférence qui assurerait la cohabitation et la synthèse entre les deux fonctions d'utilité marchande et patrimoniale élémentaires, à la manière par exemple dont Gary Becker l'a tenté à propos de ce qu'il a appelé l'économie de la famille (1981). Pour ce faire il a développé l'hypothèse d'un contenu altruiste (l'utilité des autres membres de la famille) au sein même du noyau de préférence égoïste de l'individu (spécifié comme membre de la famille - le père). La proposition d'une telle fonction de préférence est possible, dans la mesure où l'on imagine de fait abolie l'altérité des individus, ce qui revient à définir une sorte de métaindividu englobant à l'égard d'autres individus ${ }^{20}$. Ici rien de tel. L'agent économique, l'agriculteur, recouvre deux individualités porteurs de fonction d'utilité directement contradictoires l'une à l'autre. Ce qui est utilité pour l'une est désutilité pour l'autre, sans qu'il existe de règle de composition entre les deux. Ce constat de l'impasse de l'interprétation comportementaliste conduit à privilégier une approche en termes institutionnalistes, puisque dans cette démarche ce sont des institutions, éventuellement plurielles, qui imposent un comportement aux acteurs, ainsi que nous avons pu le constater à travers la famille, le groupe professionnel, se rattachant à la notion centrale de patrimoine et aux valeurs d'identité et de solidarité d'une part, ou le marché, l'entreprise, associés à des normes de comportement individualistes et concurrentiels d'autre part.

\subsection{Sur les caractéristiques d'une démarche institutionnaliste}

Il nous semble de ce point de vue qu'on ne peut éviter de s'intéresser à deux éléments clés de la démarche de Commons : la nécessité de substituer à la démarche néoclassique une approche en termes de transaction et la nécessité de prendre en compte l'action et les transformations du cadre juridique dans la dynamique économique. En effet, si l'on retient avec Hodgson $(2000,327)$ que l'institutionnalisme se définit essentiellement par son opposition à l'individualisme méthodologique en affirmant "l'idée que l'individu est socialement et institutionnellement constitué ", il reste à spécifier

20 Et c'est cette hypothèse qui a été l'objet des critiques les plus vives (Bergstrom, 1989 ; Bruce, Waldman, 1990).

Economie et Institutions $-\mathrm{n}^{\circ} 3-2^{\mathrm{e}}$ semestre 2003 
les outils analytiques permettant de rendre compte d'une telle constitution $^{21}$.

\subsubsection{La question de l'unité élémentaire d'analyse: la transaction}

L'une des premières caractéristiques de la démarche commonsienne est d'abandonner l'unité d'analyse néo-classique, la relation de l'individu aux objets rares susceptibles de satisfaire son désir, au bénéfice d'une unité inter-individuelle, la transaction. Dans l'analyse traditionnelle "les unités" élémentaires de sens " économique étaient entre l'homme et la nature, non entre l'homme et l'homme. Ainsi de la relation ricardienne entre le travail humain et la résistance des forces de la nature; ou bien de la relation de Menger entre la quantité des forces naturelles désirées et la quantité disponible" (Commons, 1934, 56). Dans ces constructions "ni le statut légal, ou éthique, ou coutumier, non plus que les décisions judiciaires " n'étaient pris en considération, " ou plutôt, tout ceci était éliminé en supposant que la propriété était identique à la matière possédée, dans la perspective de construire une théorie d'économie pure basée sur les seuls échanges physiques de matériels et de services" (id.). Un tel postulat élude en effet la question de la définition sociale de l'objet, comme on peut le voir dans l'exemple du bail rural. La tradition économique classique considère que celui-ci porte sur la mise à disposition des forces productives naturelles de la

$21 \mathrm{Si}$ les institutionnalistes partagent une "vue du monde dans laquelle le pouvoir, les conflits, les défaillances de marché et les possibilités d'une politique gouvernementale active sont plus prononcées que pour les économistes standards ", leur méthode reste mal caractérisée (Lind, 1993, 13), les divergences l'emportant sur les convergences (Alston, Vaughan, 1993). Certains mettent en avant la place accordée aux études empiriques (Fusfeld, 2000), ou le refus de la constitution d'un corps théorique unifié, signalant que la publication de l'ouvrage de Commons "Institutional Economics " (1934) créa la consternation parmi ceux qui, à l'époque, se déclaraient institutionnalistes (Rutherford, 2000, 300). Hodgson confirme ce manque d'intérêt " pour construire une théorie générale, englobant tous les aspects. Au contraire, les phénomènes complexes sont envisagés sur la base d'un nombre limité de concepts communs et d'outils théoriques spécifiques " (Hodgson, 1998, 168). Celui-ci retient cinq critères (proposition de politiques; recours aux idées et données d'autres disciplines telles que la psychologie, la sociologie et l'anthropologie ; les institutions comme élément central de toute économie ; encastrement de l'économie dans un ensemble de relations sociales, culturelles, politiques et de pouvoir ; refus de l'individu comme pré-donné et considération qu'il est fondamentalement déterminé par les institutions) ; mais il considère que seul le dernier d'entre eux permet d'établir véritablement une frontière entre les institutionnalistes et les autres économistes (Hodgson, 2000).

136 Economie et Institutions $-n^{\circ} 3-2^{e}$ semestre 2003 
terre (pour les néo-classiques il s'agit des services producteurs d'un capital qui n'est pas productible, ce qui revient au même quant à notre propos). Le présupposé naturaliste de cette position consiste à croire qu'il existe un tel objet, les forces productives naturelles ou les services de la nature, indépendamment et antérieurement à la relation d'appropriation, qui prend ici la forme de la relation entre le propriétaire et le locataire. En toute rigueur, et comme le montre l'examen de la pratique relationnelle instituée par la coutume et le droit, la définition de la fertilité du sol est indissociable de l'acte de constitution de la relation entre le propriétaire et le locataire, par où sont définies les conditions de son exploitation et de sa reproduction (Barthélemy, 1982). Supposer l'existence d'un objet naturel qui serait disponible à l'appropriation de l'individu rejoint les robinsonnades bien connues du naturalisme inhérent à l'individualisme du Siècle des Lumières et de l'économie traditionnelle. Il n'existe rien de tel, qui puisse raisonnablement apparaître comme objet premier; les objets (de l'économie) qui manifestent la rareté ou satisfont les besoins sont des construits sociaux contemporains des relations sociales dans lesquelles ils s'insèrent, à travers des normes de propriété. Dans le cas du bail rural, la fertilité "naturelle " de la terre, ou les services producteurs d'un capital foncier "non productible", sont inconcevables, même pour des agronomes, en dehors d'un processus déterminé de production, lequel n'existe pas s'il n'y a pas déjà une relation de propriété, déterminant les positions respectives du propriétaire et du locataire.

Comme le souligne Commons, l'un des avantages de l'élision des rapports de propriété "pour la théorie économique classique, basée sur les relations de l'homme à la nature, est de ne rencontrer aucun conflit d'intérêts dans ses unités d'investigation, dans la mesure où ses unités étaient les biens et les individus, la propriété étant omise. Ces unités élémentaires produisaient, en fait, et en correspondance avec l'analogie de l'équilibre, une harmonie d'intérêts plutôt qu'un conflit d'intérêts" (57). Si l'on prend au contraire en considération la réalité, il faut adopter un autre point de vue. "L'unité de base de l'activité, qui réunit le droit, l'économie et l'éthique, doit contenir en elle-même les trois principes de conflit, de dépendance et d'ordre. Cette unité est une transaction,... la plus petite unité de l'économie institutionnaliste" (58). La dépendance signifie que la propriété n'a pas de sens, de définition concevable, indépendamment de l'usage, donc que le propriétaire et le locataire se définissent mutuellement; le conflit porte sur la caractérisation des propriétés de l'objet de la relation (où commence et finit la fertilité du sol relativement à la fertilité du travail du locataire et de son capital d'exploitation, et par voie de conséquence dans quelle proportion chacun va-t-il tirer profit de l'activité définie en commun ?) ; quant à 
l'ordre, c'est celui de la coutume et du droit, qui pose à chaque période un cadre à la relation de location (Barthélemy, 1981).

A travers l'exemple du bail rural, qui occupe une place symptomatique dans notre exposé empirique, on peut percevoir une dimension générique importante de l'approche institutionnaliste, au moins au sens commonsien : l'indétermination originelle de l'objet (ici la fertilité du sol) renvoie à la nécessité d'une construction sociale de sa propriété. Dans le cas présent, au-delà des dimensions intrinsèques de cette transaction (conflit, dépendance, ordre) on remarquera qu'en fait l'opération de constitution de l'objet et de sa propriété se dédouble par la formation de deux transactions distinctes et opposées. La première est marchande, selon laquelle le fermier achèterait la fertilité du sol défini en commun, ce qui suppose une forme de propriété dans laquelle le bail lui assure une indépendance dans l'investissement en travail et capital d'exploitation, une durabilité temporelle correspondante (ou l'obligation $\mathrm{du}$ propriétaire d'indemniser si celle-ci n'est pas accordée), et la cessibilité marchande du bail en tant que moyen de production de l'entreprise qu'il établit sur l'exploitation de la fertilité du sol définie en commun. La seconde est patrimoniale, ce qui suppose la forme de propriété correspondante : la constitution d'un groupe patrimonial entre le propriétaire et le locataire pour lequel la fertilité foncière est patrimoine commun; en ce sens la relation d'interconnaissance (exclusive de l'anonymat marchand) est constitutive du bail, incluant une dimension d'association entre les famille avec la cession non vénale du bail réservée aux deux lignées, selon un ordre hiérarchique ; le locataire tire "les fruits de l'héritage affermé " sous le contrôle du propriétaire qui s'assure qu'est respectée la culture "en bon père de famille " c'est-à-dire que le fermier reconstitue à mesure la fertilité supposée naturelle du sol. Ainsi le même bail est le support de deux transactions, à travers des prescriptions normatives s'organisant en deux systèmes distincts l'un de l'autre (Barthélemy, 1982), comme on l'a illustré précédemment ${ }^{22}$. Ceci renvoie à la dimension dialectique, selon nous nécessaire, de la démarche institutionnaliste, parce que la définition d'une propriété s'opère par le déni implicite d'une autre. Nous aborderons ultérieurement cet aspect des choses, réservant pour l'instant notre attention à la notion d'institution.

22 Commons distingue trois types de transaction: d'échange, de direction, distributive (pour reprendre la traduction des termes de bargaining, managerial et rationning retenue par Bazzoli 1999). Dans notre travail nous avons mis en évidence une transaction marchande et une transaction patrimoniale. En l'état actuel de notre réflexion nous penchons pour une contextualisation du concept plutôt que pour un respect strict de la classification des transactions proposée par Commons, et sur laquelle il se justifie finalement assez peu.

$138 \quad$ Economie et Institutions $-n^{\circ} 3-2^{e}$ semestre 2003 


\subsubsection{Institution, droit, et intérêt général}

L'institution se présente, pour Commons, comme l'ensemble formé par les organisations auxquelles sont associées des règles de fonctionnement : "les trois types de transaction sont liées entre elles dans une unité d'investigation plus vaste qui est nommée organisation (going concern). Ce sont ces organisations, avec les règles de fonctionnement (working rules) qui les maintiennent en action, allant de la famille, à l'entreprise, au syndicat, jusqu'à l'Etat lui-même, que nous appellons institutions" (Commons, 1934,69). L'institution se présente comme un niveau supérieur par rapport aux transactions élémentaires: celles-ci sont reliées entre elles par les forces de l'organisation ${ }^{23}$, elle-même stabilisée par des règles de fonctionnement.

Les institutions sont une nécessité pour l'existence même de l'action individuelle: "si nous nous efforçons de trouver un principe universel, commun à tous les comportements qualifiés d'institutionnels, nous pouvons définir une institution comme une action collective en contrôle de l'action individuelle " (Commons, 1934, 69). Ce contrôle n'est pas une simple restriction: il comporte une "libération" au regard des coercitions ou discriminations qui pourraient affecter l'action individuelle. L'approche institutionnelle de Commons tisse ainsi un réseau de relations entre action individuelle et collective au sein duquel il n'est plus de sens à attribuer une valeur exclusive à l'acte personnel de l'individu isolé.

Cette imbrication est à vrai dire déjà présente dès qu'est posée la notion de transaction, avec sa triple dimension de conflit, dépendance et ordre. Les deux termes de complémentarité et conflit renvoient à la confrontation nécessaire et indéfinie de deux intérêts pris dans leur opposition, ce qui signifie un incertain compromis entre ces deux termes en tension. Aucune stabilité ou prévisibilité de relation économique ne pourrait être envisagé sans le troisième terme, la relation d'ordre. Or celle-ci fait intervenir la société comme telle, l'ensemble des individus organisés selon des règles de coexistence pacifié, même provisoirement. Ici le droit occupe une place essentielle. Comme le relève Kirat, "il faut insister sur le fait que l'unité de base de l'analyse de Commons -la transaction- est une catégorie d'emblée juridicisée: elle implique en effet non pas deux parties, comme l'économie politique tend à le poser, mais cinq: le vendeur, l'acheteur, les vendeurs alternatifs, les acheteurs alternatifs, et l'institution judiciaire" (Kirat, 2001, 15). La communauté des

23 Comme le fait remarquer Bazzoli (1999, 103-106) l'expression going concern (qui est de forme grammaticale progressive) contient les deux dimensions d'une organisation en tant que mouvement en cours, et en tant que forme fixée de mouvement.

Economie et Institutions $-n^{\circ} 3-2^{e}$ semestre 2003 
acheteurs et de celle des vendeurs est présente, spécifiant la dimension compétitive de la transaction marchande, mais la clé de voûte de l'institution au sens de Commons est le système judiciaire entendu comme l'énoncé des normes de comportement accompagné des moyens de mise en oeuvre et de sanction. Un rôle essentiel au "legal-economic nexus", expression qui désigne "l'interpénétration des relations économiques et juridiques " dans laquelle le droit apparait comme "un vecteur central de régulation des transactions économiques, en coordonnant et rendant compatibles la multiplicité des intérêts " (Bazzoli, 1999, 93).

Par cette mise en avant du complexe économico-juridique, Commons établit une rupture très sensible à l'égard des travaux des économistes non institutionnalistes. Comme il le montre à propos de la manière dont la Cour Suprême des Etats-Unis fait passer entre 1872 et 1897 la notion de valeur économique d'une définition fondée " sur la valeur d'usage des choses physiques à la valeur d'échange de toute chose " (Commons, 1924,21), le contenu des concepts est, dans les faits, objet de contestation et d'évolution, sanctionné par la reformulation périodique des énoncés juridiques. La "conception contextuelle de la signification qui est défendue " par les pragmatistes "en critique du formalisme" des économistes traditionnels (Bazzoli, 1999, 69) oblige à observer pas à pas l'évolution que font subir les acteurs au sens des concepts ; l'expérience pratique de Commons est que le système juridique occupe une place stratégique, parce qu'il stabilise périodiquement cette mouvance sémantique.

On débouche alors sur la dimension herméneutique du droit dans le complexe économico-juridique. Comme le fait remarquer Commons, les décisions de justice, et notamment de la Cour Suprême, sont toujours pris "en référence à une finalité publique" $(1934,651)$. Certaines des formulations de Commons sont certes parfois peu explicites à ce sujet. Lorsqu'il écrit par exemple que la méthode de la Common Law ${ }^{24}$ " pour faire de nouvelles lois " consiste " à retenir les coutumes changeantes de la majorité de la population à un moment donné, et à les formuler en règles d'action collective par un processus rationalisant de justification" (682), faut-il entendre une interprétation ultra-empiriste du droit et une forme d'instrumentalisation de celui-ci par les plus forts ou les plus nombreux, selon une pente commune à la vulgate marxiste et aux dérives actuelles du néo-classicisme vers les théories du lobbying politique, ou bien faut-il considérer le travail de rationalisation comme l'élément central, renvoyant à une construction rigoureuse,

24 D'une manière générale Commons survalorise la flexibilité du système jurisprudentiel de la Common Law, dévalorisant un peu abusivement les systèmes de droit romano-germaniques $(1934,714)$ qui, dans les faits, ne sont pas si différents. Surtout l'accent mis sur la flexibilité tend à masquer l'importance de la notion d'intérêt général. 
même si changeante, de l'intérêt général ? A considérer la jonction qu'établit Commons entre l'élaboration du droit moderne et la fondation du capitalisme (1924), et son "plaidoyer pour un capitalisme raisonnable " (Bazzoli, 1999, 137), il adopte le second terme de l'alternative.

Dans cette affaire le point principal est le suivant : le travail d'élaboration des règles suppose la pré-définition d'un intérêt général, ou de valeurs sociales par rapport auxquelles la (re)formulation des normes de comportement sont déduites rationnellement ou "justifiées ", selon l'expression qui sera utilisée ultérieurement dans le même sens par Boltanski et Thévenot (1987). Le travail que nous avons mené sur l'allocation des moyens de production en agriculture fait ainsi apparaitre deux principes d'intérêt général: la liberté individuelle d'entreprendre avec ses perspectives d'innovation et de croissance, qui nécessite l'ordre marchand et son régime de concurrence, la solidarité et la continuité intergénérationnelle impliquant l'établissement d'un ordre patrimonial. L'hypothèse d'une dualité de principes d'intérêt général suscite la question de leur relation ; avant de l'aborder comme telle il n'est pas inutile d'en spécifier l'enjeu pour les économistes à travers la notion de "valeur raisonnable".

\subsection{Valeur raisonnable, institution et prix}

La transaction économique se manifeste comme "un procès d'évaluation jointe par les participants, chacun étant agi par la diversité des intérêts, sa dépendance des autres, et par les règles collectives qui, à ce moment là, imposent une conformité des transactions à l'action collective. Ainsi, les valeurs raisonnables sont des transactions raisonnables, des pratiques raisonnables, et une utilité sociale, équivalente à l'intérêt général (public purpose)" (Commons, 1934, 681). Dans une pensée qui associe le droit et l'économie, "la valeur raisonnable, à un niveau abstrait, n'est rien d'autre qu'une solution cohérente et pragmatique, bien que sécularisée, au problème du juste prix" (Ramstad, 2001, 254). La solution institutionnaliste à ce problème est que "les prix sont des conventions sociales, renforcées par les habitudes et encastrées dans des institutions spécifiques" (Hodgson, 1998, 169). Ainsi, "une approche institutionnaliste de la théorie des prix commence par examiner les institutions dans lesquelles les prix sont formés " (170).

En retenant ce point de vue, l'économiste nous semble devoir être amené à établir une distinction entre valeur et prix, dans la mesure où le terme de valeur se trouve surchargé de sens pour les institutionnalistes. Il ne s'agit pas de revenir aux anciennes distinctions entre valeur naturelle (de travail) et prix de marché, ou entre valeur objective et valeur subjective, mais de se référer au 
positionnement institutionnel relatif des finalités et des normes. En ce sens, nous ne nous éloignons que modestement de Commons, pour lequel "la valeur économique a une double face, à la fois inséparable et incommensurable: c'est une grandeur monétaire mettant en rapport la valeur d'usage (quantité et qualité) et la valeur de rareté (prix)", étant bien entendu que "ce sont les transactions et les règles collectives ", "qui sont causes et régulateurs des valeurs " (Bazzoli, 1999, 124). Outre une réserve qu'il nous paraît nécessaire de poser quant à la nature nécessairement monétaire des valeurs économiques, un aspect nous paraît insuffisamment explicité : le terme de valeur, comme dans 'valeur raisonnable' désigne effectivement le résultat de l'action des transactions et règles collectives; mais par ailleurs il désigne aussi bien la force déterminante des institutions, puisque les intérêts particuliers qui se manifestent dans les transactions élémentaires au sein de celles-ci doivent se confronter à l'arbitrage rendu au nom de l'intérêt général, c'est-à-dire une valeur préexistante à celle qui sera résultat.

Les institutions comme systèmes de règles collectives d'action sont à concevoir, comme on l'a vu, dans une perspective de justification rationnelle, ce qui implique des principes de constitution interne en cohérence avec certaines finalités ${ }^{25}$. Cette utilité sociale, ou intérêt général, à laquelle se réfèrent les transactions et les normes de pratique qui leur sont associées, se conjugue différemment selon les entités considérées, sauf à supposer que les diverses institutions auxquelles Commons faisait par exemple allusion, la famille, l'entreprise, le syndicat, l'Etat, se définissent en référence à des finalités sociales identiques, ce qui priverait de sens la distinction entre institutions. La description d'une institution, au sens de Commons, passe donc par celle de l'utilité sociale, ou du noyau d'utilités sociales cohérentes entre elles, autour de laquelle s'organisent les règles de comportement spécifique à cette institution. A ce niveau d'expression, qui est celui de la notion de valeur "cause et régulatrice", on est en symbiose avec la notion de valeur sociale telle qu'elle est utilisée en sociologie pour décrire le référentiel des pratiques comportementales.

Il nous semble nécessaire pour les économistes, sans vouloir entrer dans un débat sur la définition et le champ de la science économique mais dans le simple souci de respecter l'étymologie du terme qui désigne notre pratique scientifique, d'adjoindre aux valeurs ainsi définie la question spécifique des prix. Les transactions, au sens économique, portent sur la propriété des biens, par où ces biens sont échangés, produits, répartis. Chaque catégorie de

25 Ici la notion de finalité, et de rationalité qui lui est associée en tant qu'ajustement spécifique des normes en vue de satisfaire la finalité, est plus à entendre comme nécessité fonctionnelle de cohérence entre les parties et le tout, que comme a priori téléologique.

$142 \quad$ Economie et Institutions $-n^{\circ} 3-2^{e}$ semestre 2003 
transaction avec ses corps de règles implique des mouvements de propriété de biens en des quantités nécessairement définies, sinon il n'y aurait pas d'ordre. Que la valeur raisonnable soit issue d'un processus de conciliation d'intérêts opposés, sous l'égide d'un ordre juridique énoncé en référence aux valeurs sociales spécifiques de l'institution considérée, ne pallie pas mais au contraire implique, du point de vue économique, que ce processus conduise à une formation de prix, c'est-à-dire des classes d'équivalence entre les quantités de biens qui seront finalement échangés, produits ou répartis. En ce sens, la spécificité de l'économie institutionnaliste, en tant qu'elle se différencie des institutionnalismes sociologiques ou historiques, serait de mettre en évidence les règles de formation des prix spécifiques à chacune des institutions concernées. Tel est en tout cas la nécessité analytique que nous nous sommes posée dans l'étude de l'allocation des moyens de production en agriculture, dont le résultat a été la mise en évidence de deux modes spécifiques de détermination des prix en tant que classes d'équivalence entre quantités de biens associées l'une à l'ordre marchand et l'autre à l'ordre patrimonial.

Une telle considération de la nécessité, par les économistes, d'associer à la définition d'une institution particulière, outre l'utilité sociale qui la caractérise, celle de son mode spécifique de détermination des prix ouvre la porte à une nouvelle interrogation. D’une manière générale, les économistes standard, tout à leur projet normatif, nient le problème de la pluralité des systèmes de prix en se donnant pour tâche de ramener tout prix à la catégorie marchande quand bien même les apparences en seraient directement contraires (Barthélemy, Nieddu, Vivien, 2004). Dès lors que les économistes institutionnalistes reconnaissent la pluralité des catégories de prix, chacune d'elle rattachée à la définition d'une institution particulière, le problème analytique devient plus complexe. Concevoir la conciliation des systèmes de valeur, et des systèmes de prix, relevant d'institutions distinctes entre elles suppose qu'on dispose d'une démarche qui ait pour souci de contextualiser les effets de composition entre valeurs différentes, tout en rendant compte des dynamiques produites par de tels effets. On se permettra de revenir ici aux propositions méthodologiques contenues dans un texte déjà ancien (Wilber et Harrison, 1978), mais toujours d'actualité de notre point de vue.

\subsection{Concepts contextualisés et dialectique}

Wilber et Harrison considèrent que "...l'implicite du travail des institutionnalistes est un 'mode d'investigation' commun (...) qui se conforme à ce que les philosophes des sciences appellent un modèle d'interrelation (pattern model)" (Wilber et Harrison (1978, p. 73). Ce 
modèle d'interrelation nous intéresse à double titre : d'abord parce qu'il propose d'organiser à travers des récits (storytelling) la restitution de l'unité d'articulation des parties dans un tout qui les transcende; ensuite parce qu'ils proposent d'utiliser l'organisation du récit pour rendre compte de la dimension dialectique de rencontre entre des éléments contraires qui ne serait pas visible sans leur mise en scène.

\subsubsection{La dialectique pour identifier des concepts contextualisés?}

De ce point de vue divers auteurs qualifient la démarche de Commons de holiste, même si l'usage du terme n'était pas répandu à l'époque (Ramstad, 1986 ; Biddle, Samuels, 1994). Par là, il faut entendre que "la réalité est conceptualisée comme un tout intégré, une unité, et non comme un ensemble de structures et processus logiquement séparables" (Ramstad, op.cit., 1071). L'objectif " à obtenir en économie est d'exposer une théorie $d u$ processus scientifique qui conduit à une compréhension de la relation entre partie et totalité " (Commons, 1934, p.734-735). Il en découle que le travail d'élaboration holiste comprend un exercice d'exposition des différents aspects d'une même réalité -qui se présente comme un récit organisé (storytelling)-, qui conduit à la proposition "d'un modèle d'interrelation. Il est construit en liant les hypothèses ou thèmes validés en un réseau ou modèle" (Wilber, Harrison, 1978, p.76). ${ }^{26}$

Quant aux caractéristiques de l'élaboration, Wilber et Harrison soulignent que " pour user de la terminologie de Kaplan, la structure des théories holistes est concaténée ${ }^{27}$ plus que hiérarchique " (idem). La structure concaténée du "storytelling " institutionnaliste est rendue nécessaire par sa conception holiste de la réalité : un élément du système ne peut être compris comme le reflet d'un principe universel, ou comme une partie fonctionnelle d'un tout organisé de façon hiérarchique et unidimensionnnelle. Par exemple, dans notre "storytelling " des modalités d'allocation des moyens de

26 Le point de vue holiste, en référence à la pensée pragmatiste, est que ce type d'analyse "est correcte si, à mesure que le modèle devient plus complexe et détaillé, une plus grande diversité des données trouve aisément sa place. A ce stade, il devient plus difficile d'imaginer un modèle alternatif ou une explication qui réussisse à inclure les mêmes thèmes. En conséquence, l'explication du système dans son entier est tenue pour vraie, jusqu'à ce qu'un modèle alternatif ou révisé soit capable de surpasser l'ancien modèle en intégrant une diversité toujours plus grande de données "(idem, p.77).

27 On entend par concaténation, le fait qu'il faille disposer d'un enchaînement des causes et des effets, tel qu'on n'ait plus aucune proposition étrangère sous-entendue.

144 Economie et Institutions $-n^{\circ} 3-2^{e}$ semestre 2003 
production de l'agriculture, aucune règle n'est considérée en ellemême comme ensemble fonctionnel. Ainsi des normes portent sur la dimension d'exploitation, mais certains de leurs alinéas se rattachent à la promotion de l'entreprise et du marché en agriculture (seuil minimum de viabilité) et d'autres à la défense de la construction patrimoniale (seuil maximal et d'agrandissement); de même le bail rural, par ses règles relatives à l'investissement favorise l'entreprise, tandis que par celles sur la cession, il avantage l'interconnaissance et la famille, et donc la dimension patrimoniale. Chaque alinéa se rattache de fait à deux institutions différentes, sans que soit posée la nécessité qu'elles soient cohérentes entre elles.

C'est à cet endroit, nous semble-t-il que trois aspects de la démarche dialectique peuvent être le support d'une démarche de recherche. D'abord, l'élaboration d'un concept contextualisé tel que celui correspondant à une institution précise fait apparaître "qu'un concept opposé a été implicitement dénié par le premier "; ensuite on découvre que "le concept opposé est nécessaire à la validation ou l'acceptabilité du premier" ; enfin le problème théorique réel est " celui de l'interrelation entre les deux" (Wiber et Harrison, op. cit. p.82). Cette proposition selon laquelle le holisme inclurait une dimension dialectique est particulièrement intéressante dans la mesure où l'on trouve là une des rares approches explicites de la question théorique des relations entre institutions : si chacune d'elle établit son propre système de normes, en dehors de toute hiérarchie présupposée, et qu'elle se présente comme autosuffisante, à partir de quel concept penser la cohérence d'ensemble, cette unité postulée sous le terme même de holisme?

\subsubsection{Quand est-on en présence d'une institution ?}

Lorsqu'on parle de cohérence d'ensemble, il ne s'agit pas de renvoyer à la stabilité fonctionnelle d'un système, mais aux mouvements qui l'animent: on rejoint ici l'idée défendue par Commons, selon laquelle l'évolution est indéfinie: "Nous partons, comme le fait Veblen, à un croisement, à un point du temps, d'un processus qui n'a ni début ni fin" (Commons, 1934, p. 678). Et "c'est dans le changement de ces règles collectives, incluant la coutume et les organisations, et toutes sortes de philosophies sociales, que nous trouvons, comme le fait Veblen, la théorie évolutionniste de l'économie" (idem, p. 656). S’il est donc clair que, pour Commons, l'évolution n'est pas autre chose que le mouvement des institutions, dans toutes leurs composantes de valeurs sociales, normes et organisations, reste cependant posée la question de savoir comment on sépare analytiquement institutions et organisations. Comment 
reconnaît-on que l'on est en présence d'une institution et non d'un organisation collective?

Comme pour toute recherche, notre exposé de la première partie présente le résultat de la recherche et non la démarche de recherche elle-même, avec ses échafaudages. Si l'on se place à un niveau méthodologique, notre premier pas a été de s'interroger sur ces résidus au développement du marché, si tenaces et consistants qu'ils font depuis le $19^{\circ}$ siècle l'objet de "la question agraire " et de la vindicte des économistes du marché. La résistance à la marchandisation de la terre, les restrictions à son usage, les règles de répartition familiales bloquant l'accès aux règles marchandes... tous ces éléments non intégrables dans une pensée du marché n'étaient-ils que des restes disparates ou ne pouvaient-ils trouver place dans un autre concept "implicitement dénié", selon une cohérence dont la plausibilité serait à tester ? Ainsi est apparue l'hypothèse d'une logique patrimoniale, et en même temps, pour que cette conception soit possible, le mouvement. En effet, le marché intervient dans une opération de destruction de l'ordre patrimonial préexistant, avec ses valeurs d'interconnaissance et de solidarité. Il ne s'agit pas seulement du jeu d'intérêts privés, mais il s'agit explicitement de proposer de nouvelles valeurs "d'intérêt général ": l'ouverture aux échanges, la croissance grâce à la force de la compétition.

Or, si le mouvement était seulement contenu dans cette opération de destruction liée par la tentative de l'ordre marchand de supplanter une institution par une autre, tout s'arrêterait avec le succès de cette opération, et le passage d'une agriculture familiale à une agriculture d'entreprise. La thèse que nous avons défendue est que l'institution patrimoniale, dans sa logique intrinsèque, persiste au delà des changements d'organisation. L'institution patrimoniale en matière de production agricole change de forme, de la famille au groupe professionnel parce que l'organisation familiale ne suffit pas à résister à la poussée du marché : il ne s'agit, au sens de Commons que d'organisations (going concern), et non d'institutions, car ce qui est continu, ce sont les valeurs sociales, "l'intérêt général" que pourrait porter l'interconnaissance, la solidarité de groupe, et le caractère non marchand des règles d'allocation par où s'accomplissent ces finalités. De ce point de vue, l'institution ne peut être assimilée à la forme phénoménale qui porte à un moment donné sa logique. Dans la méthode abductive que nous défendons, l'institution ne peut donc que se situer au niveau d'abstraction de la logique qu'elle promeut.

De façon générale, nous avons montré ailleurs (Barthélemy, Nieddu, Vivien, 2004), que la relation marchande suppose que les acteurs s'abstraient des autres relations sociales pour atteindre à l'anonymat nécessaire à la réalisation de la transaction. En cela, s'il 
est possible à des économistes de présenter des modèles de sociétés ou les agents ne sont reliés que par des relations marchandes, il ne peut s'agir que d'exercices de style. C'est à cet endroit que le principe de réalisme commande de considérer la nécessité pour un fonctionnement social effectif de maintenir des principes de solidarité entre les mêmes acteurs de la compétition marchande et donc de rechercher, du point de vue de l'économiste, la persistance de l'institution patrimoniale. Celle-ci, au delà des changements d'organisation, marque sa présence par l'altération qu'elle provoque dans les formes des règles marchandes. C'est, selon nous ce qui explique le fait que le débat social soit traversé par des oxymores (" capitalisme raisonnable ") ou des " concepts mous " parfois traités avec mépris par les économistes professionnels comme trop journalistiques: les notions de "juste prix ", de "valeur raisonnable ", ou dans les domaines empiriques qui nous occupent de "développement durable " et de "multifonctionnalité de l'agriculture".

C'est bien à cet endroit qu'on peut percevoir l'intérêt d'une approche institutionnaliste dialectique, qui ne nie pas l'action modificatrice des individus et des groupes, puisque c'est la tension entre référentiels contradictoires qui pousse les acteurs à l'innovation institutionnelle. Elle propose un cadre d'interprétation qui signale que toute action, toute modification dans l'organisation du marché impliquera une réaction d'ajustement de l'organisation patrimoniale. En même temps, la notion de l'unité d'intégration, au centre de la démarche holiste, se trouve éclairée. Celle-ci n'est effectivement pas à concevoir comme étant elle-même une institution, car la tension contradictoire entre marché et solidarité serait réglée d'avance par une relation d'ordre, mais comme l'ensemble des deux institutions marchandes et patrimoniales mises en mouvement par leur jeu réciproque d'antagonisme et de dépendance.

\section{REFERENCES BIBLIOGRAPHIQUES}

Alston, R.M., Vaughan, M.B., (1993), "Institutionalists: A United Front or Divergent Voices of Dissent?", Journal of Economic Issues, vol.27, 2, June, 351-361

Augustins, (1989) Comment se perpétuer? Devenir des lignées et destins des patrimoines dans les paysanneries européennes, Nanterre, Société d'Ethnologie, 434 p.

Barrere, C., Barthelemy, D., Nieddu, M., Vivien, F.D, (eds), (à paraitre 2004), Repenser le patrimoine, Paris, L'Harmattan

Barthelemy, D., (1981), "Le cycle du capital foncier", Revue d'Economie Politique, $\mathrm{n}^{\circ} 4$, juil.-août, pp. 349-372 
Barthelemy, D., (1982), Propriété foncière et fonds-entreprise. La production du capital foncier en agriculture, Paris, Economica, 190 p.

Barthelemy, D., (1988), La naissance de l'entreprise agricole, Paris, Economica, 184 p.

Barthelemy, D., (2002), "Economie patrimoniale et répartition des moyens de production dans l'agriculture française ", Economie Rurale, 268-269 : 89-102

Barthelemy, D., Boinon, J.P., (1973), "Le caractère familial du développement des exploitations agricoles du Châtillonnais ", Dijon, INRA, Série Doc. de Recherches (4), mars, 74 p.

Barthelemy, D., David, J., (éds.), (2001), Production Rights in European Agriculture Paris, Amsterdam INRA Elsevier, $245 \mathrm{p}$

Barthelemy, D., Nieddu, M., Vivien, F.D., (à paraître 2004), "Economie patrimoniale, identité et marché ", in, Barrere, C., Barthelemy, D., Nieddu, M., Vivien, F.D, (eds), Repenser le patrimoine, Paris, L'Harmattan

Bazzoli, L., (1999), L'économie politique de John R. Commons - Essai sur l'institutionnalisme en sciences sociales, L'Harmattan, Coll. Etudes d'économie politique, $234 \mathrm{p}$.

Bazzoli, L., Kirat, T., (1997), "Deux regards non hayekiens sur l'efficience et la sélection des règles juridiques", Archives de Philosophie du Droit, $\mathrm{n}^{\circ} 42$, pp. 257-283

Becker, G.S., (1981), A Treatise on the Family, Harvard University Press, $288 \mathrm{p}$.

Bergmann, D., Baudin, P., (1989), Politiques d'avenir pour l'Europe agricole, Paris, INRA-Economica, $170 \mathrm{p}$.

Bergstrom, T.C., (1989), "A Fresh Look at the Rotten-Kid Theorem and Other Household Mysteries", Journal of Political Economy, vol 97, $\mathrm{n}^{\circ} 5$, pp 1138-1159

Berriet-Solliec, M., Boinon, J.P., (2002) "Installation et accès au foncier à travers les projets agricoles départementaux et le fonctionnement de quelques Commissions Départementales d'Orientation de l'Agriculture ", Actes du colloque L'accès au métier d'agriculteur CNASEA Toulouse, 5 et 6 mars pp 67-72

Biddle, J.E., Samuels, W.J., (1994), "Introduction to the Transaction Edition", in COMMONS, J.R., [1924], Legal Foundations of Capitalism, The Mac Millan Company, réédition 1995, Transaction Publishers, pp.ix-xxxiii.

Blanc, M., Perrier-Cornet, P., (1993), "Farm transfer and farm entry in the European Community", Sociologia Ruralis, 33, 3-4, 319-335

Boltanski, L., Thevenot, L., (1987), Les économies de la grandeur, Cahiers du centre d'études et de l'emploi, n³1, PUF, Paris, 361p.

Brossier, J., Chia, E., Marshall, E., Petit, M., (1997), Gestion de l'exploitation agricole familiale. Eléments théoriques et méthodologiques, Dijon, ENESAD-CNERTA, 215 p. 
Bruce, N., Waldman, M., (1990), "The Rotten-Kid Theorem meets the Samaritan's Dilemman, The Quarterly Journal of Economics, February, pp 155-165

Caillavet, F., Guyomard, H., Lifran, R., (eds), (1994), Agricultural household modelling and family economics, Elsevier, Amsterdam, $326 \mathrm{p}$.

Chombart de Lauwe, J., Poitevin, J., Tirel, C., (1969), Nouvelle gestion des exploitations agricoles, Dunod, Paris

Commons, J.R., (1995) [1924], Legal Foundations of Capitalism, The Mac Millan Company, Transaction Publishers, 394 p.

Commons, J.R., (1990) [1934], Institutional Economics. Its place in Political Economy, The Mac Millan Company, Transaction Publishers, vol $1 \& 2$

Dauce, P., Le Hy, A., Perrier-Cornet, P., (1993), "Transmission des exploitations agricoles et installation des agriculteurs dans la Communauté Européenne ", Actes et communications, n¹1, INRA, Economie et Sociologie Rurales, 232 p.

David, J., (1986), La transmission des exploitations agricoles en Europe Occidentale, Poitiers, ILER, 328 p.

Fusfeld, D.R., (2000), "A Manifesto for Institutional Economics", Journal of Economic Issues, vol.34, 2 June, 257-265

Fustel De Coulanges, N.D., (1984) [1864], La cité antique, Flammarions-Champs, $494 \mathrm{p}$.

Gaignette, A., Nieddu, M., (1994), "Concurrence dans le système agro-alimentaire français contemporain et formation des revenus agricoles ", Economie Rurale 220-221, mars-juin, pp.16-21.

Gasson, R., Errington, A., (1993), The farm family business, Oxon, CAB International, $290 \mathrm{p}$.

Georgescu-Roegen, N., (1965), "The Institutional Aspects of Peasant Communities ", in Energy and Economic Myths, Institutional and Analytical Ecomics Essays (1976), Pergamon Press, pp. 199-231

Gervais, M., Servolin, C., Weil, J., (1965), Une France sans paysans, Paris, Ed. du Seuil

Guillemin H. (1998) "Aux sources de l'institutionnalisme. fondements philosophiques et méthodologiques", Cahiers $d u$ CERAS, univ Reims, $\mathrm{n}^{\circ} 30$.

Hodgson, G.M., (1998), "The Approach of Institutional Economics", Journal of Economic Litterature, vol 36, March, pp ; 166-192

Hodgson, G.M., (2000), "What is the Essence of Institutional Economics "? , Journal of Economic Issues, vol.34, 2, June, 317329

Kirat, T., (2001), "Le pragmatisme, l'économie et l'intelligence des règles juridiques : leçons de la méthode institutionnaliste de J.R. Commons ", Revue interdisciplinairte d'études juridiques, ${ }^{\circ} 47, \mathrm{pp}$ $1-22$ 
Lind, H., (1993), "The Myth of Institutionalist Method", Journal of Economic Issues, vol.27, 1, March, 1-17

Mahe, L.P., Ortalo-Magne, F., (2001), Politique agricole, un modèle européen, Presses de Sciences-Po, 235p.

Morel, S, (1999), " De l'assurance chômage à l'assistance chômage, la dégradation des situations ", in Revue de l'IRES, Paris, n³0, $1999 / 2$

Nieddu M., (en coll. avec A. Gaignette), (2001), "L'agriculture française entre logiques sectorielles et territoriales (1960-1985)" in Cahiers d'économie et de sociologie rurales $n^{\circ} 37$.

Nieddu, M.., (2002), "La multifonctionnalité agricole entre marché et externalités ", in Economie Appliquée, tome LV, 2002, n 1, p.105132.

Papillon, J.C., (1995), Economie de l'entreprise, Paris, Litec.

Perrier-Cornet, P., (1997), "La transmission des exploitations agricoles et l'installation des agriculteurs en France et dans l'Union européenne ", in Guillemin, M., (comp.) Agriculture et patrimoine : une dynamique pour l'agriculture, pp. 7-28

Polanyi, K., (1983) [1944], La grande transformation, Paris, Gallimard, $419 \mathrm{p}$.

Pondaven, C., (1989), La théorie de la réglementation, efficacité économique ou efficacité politique? Application économétrique à la P.A.C., Paris, LGDJ, 408 p.

Ramstad, Y., (1986), "A Pragmatic's Quest for Holistic Knowledge: The Scintific Methodology of John R. Commons", Journal of Economic Issues, ${ }^{\circ} 4$, Dec., pp. 1067-1105

Ramstad, Y., (2001), "Johns R. Commons's Reasonable Value and the Problem of Just Price", Journal of Economic Issues, n², June, pp. 253-277

Roussel, P.A., (2001), " Non-market equivalence between production right in France" in Barthelemy, D. (éd.), David, J. (éd.), Production Rights in European Agriculture Paris, Amsterdam INRA Elsevier $\mathrm{p}$ 185-196

Rutherford, M., (2000), "Institutionalism Between the Wars", Journal of Economic Issues, vol. 34, 2, June, 291-303

Tracy, M., (1994), "The spirit of Stresa", European Review of Agricultural Economics, 21, 357-374

Wilber, C.K., Harrison, R.S., (1978), "The Methodological Basis of Institutional Economics : Pattern Model, Storytelling and Holism", Journal of Economic Issues, 12, March, pp 61-89 